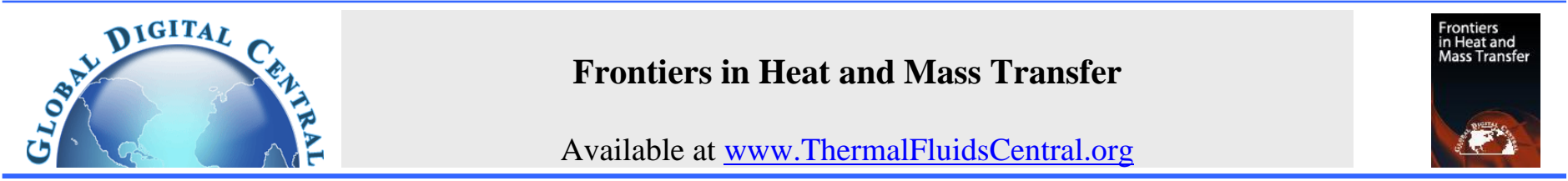

\title{
CHEMICAL REACTION AND RADIATION EFFECTS ON UNSTEADY MHD MICROPOLAR FLUID FLOW OVER A VERTICAL PLATE WITH VARIABLE TEMPERATURE
}

\author{
Mekonnen Shiferaw Ayano* and J. S. Mathunjwa
}

Department of Mathematics, University of Swaziland, Swaziland

\begin{abstract}
This paper presents a study of the Magnetohydrodynamic flow of incompressible micropolar fluid past an infinite vertical porous plate with combined heat and mass transfer. The plate oscillate harmonically in its own plane and the temperature raised linearly with respect to time. Numerical calculations are carried out for different values of dimensionless parameters and an analysis of the results shown graphically and in table form. It is found that velocity and microrotation influenced appreciatively with parameters like radiation, magnetic, chemical reaction and coupling numbers. It is also noted that microrotation highly influenced by the magnetic parameters. The effects of some parameters on the skin friction, Nusselt and Sherwood numbers studied.

Keywords: Heat and mass transfer, chemical reaction, radiation.
\end{abstract}

\section{INTRODUCTION}

Heat and mass transfer problems with chemical reaction and radiation effects gained considerable attention because of its applications. Heat and mass transfer occurs simultaneously in many applications in processes such as drying, distribution of temperature and moisture over agricultural fields and graves of fruit trees and so on. The problems of steady or unsteady flow over a vertical plate with and without chemical reaction effects are of great technical interest. Such types of problem gain considerable interest in the scientific literature to mention few: Soundalgekar and Wavre (1997) studied unsteady free convection flow past an infinite vertical plate with constant suction and mass transfer when the plate temperature oscillates in time about a constant mean. Rahman and Mulolani (2000) examines natural convection flow in the presence of both chemical reaction and diffusion the resulting flow patterns with particular interest is the observed flow reversal for higher Schmidt numbers. Un steady free convective heat and mass transfer flow through a porous medium with constant heat and mass flux and periodic permeability in slip flow regime for three dimensional case studied by Jain et al. (2012). Natural convective heat transfer from narrow vertical plates which have a uniform surface heat flux has studied with different edge conditions with special attention on Prandtl number examined by Patrick and Paul (2010). The simultaneous effects of heat and mass transfer, is concerned with a numerical study of transient natural convection flow past an impulsively started semiinfinite vertical plate subjected to uniform heat and diffusion of a chemically reactive species examined by Muthucumaraswamy and $\mathrm{P}$ (2002). Hossain et al. (2001) studied the influence of fluctuating surface temperature and concentration on natural convection flow from a vertical flat plate. Srinivasacharya and Kaladhar (2014) studied numerically us- ing Keller box method the effects of thermal and soutal stratification on mixed convection flow along a vertical plate saturated with couple stress fluid.

When the flow is under the influence of transverse magnetic field studies shoed that it has engineering applications. Chaudhary and Jha (2008)studied MHD heat and mass transfer effects on the unsteady flow of a micropolar fluid through a porous medium bounded by a semi-infinite vertical plate in a slip-flow regime taking into account a homogeneous chemical reaction of the first order. Ahmed et al. (2012) studied the effect of the transverse magnetic field, Grashof number, Prandtl number and Schmidt number on the flow and transport characteristics for an infinite vertical porous plate with chemical reaction in presence of a heat source. Hsiao (2010) examine heat and mass mixed convection for MHD visco-elastic fluid past a stretching sheet with ohmic dissipation. Muthucumaraswamy and Meenakshisundaram (2006) studied chemical reaction effects on vertical oscillating plate with variable temperature for viscous fluids.

The studies in which the fluid is Newtonian do not give satisfactory result if the fluid is a mixture of heterogeneous. A micropolar fluid introduced by Eringen (1966) contains rotating micro-constituents that cause the fluid to exhibit non-Newtonian behaviour. Micropolar fluid models have been found useful in the study of flows of exotic lubricants, colloidal suspensions, polymeric fluids, liquid crystals, additive suspensions, body fluids, turbulent shear flows and flows in capillaries and microchannels. An excellent review of micropolar fluids and their applications were provided by Ariman et al. (1990). The present study concerns the combined effects of chemical reaction and radiation on MHD flow on the velocity,

${ }^{*}$ Corresponding author. Email: mekk_aya@yahoo.com or msayano@uniswa.sz 
temperature and concentration field of micropolar fluid over the vertical plate with varying temperature. Simulation of the flow and discussion is provided for the effect of various parameters on the velocity, microrotation, temperature and concentration.

\section{MATHEMATICAL FORMULATION}

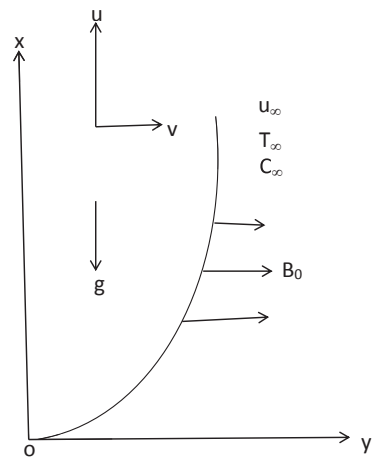

Fig. 1 Physical Model

Consider unsteady convective micropolar fluid flow with chemical reaction directly proportional to concentration (first order) and radiative heat transfer infinite vertical plate with variable temperature. The flow is assumed to be in the $x$ - direction, which is taken along the vertical porous plate and $y-$ axis axis is taken normal to the plate. Initially, the plate and the fluid are of the same temperature $T_{\infty}$ and concentration $C_{\infty}$. At time $t$ the plate starts oscillating in its own plane with frequency $\omega$ and the temperature of the plate is raised linearly with respect to time while the concentration level near the plate is raised to $C_{w}$. The fluid is assumed to be electrically conducting and variations due to temperature are limited to density and viscosity. The density variation and the effects of the buoyancy are taken into account using Boussinesq's approximation. A uniform magnetic field of magnitude $B_{0}$ is applied normal to the plate and the magnetic Reynolds number are assumed to be very small, so that the induced magnetic field is negligible. The physical model of the problem is shown in Fig. 1. The concentration of the diffusing species in the binary mixture is assumed to be very small in comparison with the other chemical species, which are present, and hence the Soret and Dufour effects are negligible. Under these assumptions the equation governing the flow of incompressible micropolar fluid is given by

$$
\begin{array}{r}
\frac{\partial v}{\partial y}=0 \\
\rho \frac{\partial u}{\partial t}+\rho v_{0} \frac{\partial u}{\partial y}=\rho g\left[\beta_{T}\left(T-T_{\infty}\right)+\beta_{C}\left(C-C_{\infty}\right)\right] \\
+\mu \frac{\partial^{2} u}{\partial y^{2}}+\kappa \frac{\partial \Gamma}{\partial y}-\sigma \beta_{0}^{2} u \\
\left.\frac{\partial T}{\partial t}+v_{0} \frac{\partial T}{\partial y}=\alpha \frac{\partial \Gamma}{\partial t}+v_{0} \frac{\partial \Gamma}{\partial y}\right)=-\kappa \frac{\partial u}{\partial y}-\kappa \Gamma+\gamma \frac{\partial^{2} \Gamma}{\partial y^{2}} \\
\frac{2 \mu+\kappa}{\rho C_{p}}\left(\frac{\partial u}{\partial y}\right)^{2}+\gamma\left(\frac{\partial \Gamma}{\partial y}\right)^{2} \\
+2 \frac{\kappa}{\rho C_{p}} \Gamma \frac{\partial u}{\partial y}-\frac{1}{\rho C_{p}} \frac{\partial q_{r}}{\partial y} \\
\frac{\partial C}{\partial t}+v_{0} \frac{\partial C}{\partial y}=D \frac{\partial^{2} C}{\partial y^{2}}-K_{1} C
\end{array}
$$

where $\rho$ is density, $u$ is fluid velocity, $T$ is temperature, $C$ concentration, $\mu$ the dynamic viscosity, $g$ is gravitational acceleration, $\beta_{T}, \beta_{c}$ are the thermal and concentration expansion coefficient, $\alpha$ the fluid thermal diffusivity, $\sigma$ fluid electrical conductivity, $K_{1}$ chemical reaction parameter, $B_{0}$ magnetic field intensity and $D$ the effective diffusive coefficient. Initial conditions:

$u(y, 0)=0, \quad \Gamma(y, 0)=0, \quad T(y, 0)=T_{\infty}, \quad C(y, 0)=C_{\infty}$ for all $\mathrm{y}$

The boundary conditions:

$$
\begin{array}{r}
\text { for } t>0: u(0, t)=v_{0} \cos \omega t, \quad \Gamma=n \frac{\partial \Gamma}{\partial y}, \\
T(0, t)=T_{\infty}+\left(T_{w}-T_{\infty}\right) A t, \quad C(0, t)=C_{w} \\
\text { and } u=0, \quad \Gamma=0, \quad T=0, \quad C=0, \quad y \rightarrow \infty
\end{array}
$$

where $n=0$ the case microelements close to the boundary stick to the wall and $n=1$ correspond the turbulent boundary layer. In Eq (4) the radiative heat flux under Rosseland approximation given by

$$
q_{r}=-\frac{\sigma^{*}}{3 k} \frac{\partial T^{4}}{\partial y}
$$

where $\sigma *$ is StephanBoltzmann constant and $k$ is mean absorption coefficient, and we assume the Eckert number to be small $<<1$.

If the temperature differences within the flow are sufficiently small, $T^{4}$ may be expanded by Taylor series. Hence, expanding $T^{4}$ about $T_{\infty}$ and neglecting higher order terms we get

$$
T^{4} \cong T_{\infty}^{4}+4\left(T-T_{\infty}\right) T_{\infty}^{3}=4 T_{\infty}^{3} T-3 T_{\infty}^{3}
$$

Substituting in $\mathrm{Eq}(9)$ into $\mathrm{Eq}(4)$ we get

$$
\begin{aligned}
\frac{\partial T}{\partial t}+v_{0} \frac{\partial T}{\partial y}=\alpha \frac{\partial^{2} T}{\partial y^{2}} & -\frac{2 \mu+\kappa}{\rho C_{p}}\left(\frac{\partial u}{\partial y}\right)^{2}+\gamma\left(\frac{\partial \Gamma}{\partial y}\right)^{2} \\
& +2 \frac{\kappa}{\rho C_{p}} \Gamma \frac{\partial u}{\partial y}-\frac{16 \sigma *}{3 k \rho C_{p}} \frac{\partial q_{r}}{\partial y}
\end{aligned}
$$

Introduce the non dimensional variables through

$$
\begin{array}{r}
\hat{t}=\frac{t V_{0}^{2}}{\nu}, \hat{u}=\frac{u}{U_{0}}, \quad \hat{\Gamma}=\frac{\Gamma}{U_{0} V_{0}}, \quad \theta=\frac{T-T_{w}}{T_{w}-T_{\infty}}, \\
\phi=\frac{C-C_{w}}{C_{w}-C_{\infty}}, \quad \hat{y}=\frac{y}{\nu} \operatorname{Pr}=\frac{\mu C_{p}}{k}, \quad M=\frac{\sigma B_{0}^{2} \nu}{\rho V_{0}^{2}}, \\
A=\frac{V_{0}^{2}}{\nu}, \quad S c=\frac{\nu}{D}, K=\frac{k_{1} \nu}{V_{0}^{2}}, \quad G r=\frac{g \beta_{T} \nu\left(T_{w}-T_{\infty}\right)}{V_{0}^{2} U_{0}}, \\
G c=\frac{g \beta_{C} \nu\left(C_{w}-C_{\infty}\right)}{V_{0}^{2} U_{0}}, \quad R a=\frac{4 \sigma^{*} T_{\infty}^{3}}{k}, \quad \hat{\omega}=\frac{\omega \nu}{V_{0}^{2}}
\end{array}
$$

Substituting equation (11) into equations (2-5), after dropping the hat we get the following non-dimensional equations

$$
\begin{array}{r}
\frac{\partial u}{\partial t}+\frac{\partial u}{\partial y}=G r \theta+G c \phi+\frac{1}{1-N} \frac{\partial^{2} u}{\partial y^{2}}+\frac{N}{1-N} \frac{\partial \Gamma}{\partial y}-M u \\
N_{1}\left(\frac{\partial \Gamma}{\partial t}+\frac{\partial \Gamma}{\partial y}\right)=-\frac{N}{1-N}\left(\frac{\partial u}{\partial y}+2 \Gamma\right)+N_{2} \frac{\partial^{2} \Gamma}{\partial y^{2}} \\
\frac{\partial \theta}{\partial t}+\frac{\partial \theta}{\partial y}=\frac{1}{P r}\left(1+\frac{4 R a}{3}\right) \frac{\partial^{2} \theta}{\partial y^{2}}+\frac{2-N}{1-N} E c\left(\frac{\partial u}{\partial y}\right)^{2} \\
+N_{2} E c\left(\frac{\partial \Gamma}{\partial y}\right)^{2}+\frac{2 N}{1-N} E c \Gamma \frac{\partial u}{\partial y} \\
\frac{\partial \phi}{\partial t}+\frac{\partial \phi}{\partial y}=\frac{1}{S c} \frac{\partial^{2} \phi}{\partial y^{2}}-K \phi
\end{array}
$$

Where $G_{r}$ is Grashof number, $M$ is magnetic parameter, $p_{r}$ is Prandtl number, $G_{c}$ is Modified Grashof number, $K$ is chemical reaction parameter, $R a$ is radiation parameter $N$ is coupling number, $N_{1}, N_{2}$ are micropolar fluid constants, $S c$ Schmidt number and $E c$ is Eckert number. Initial conditions:

$$
u(y, 0)=\theta(y, 0)=\phi(y, 0)=0
$$




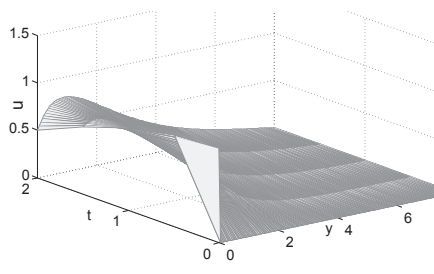

(a)

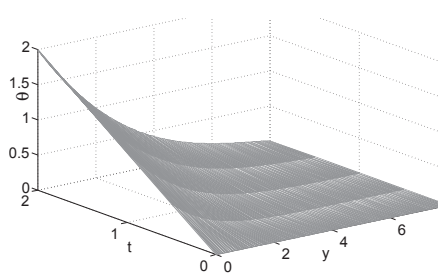

(c) (b)

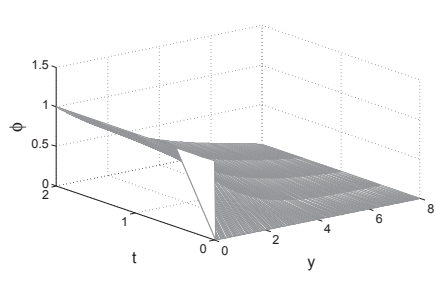

(d)

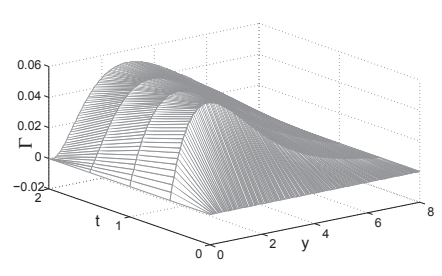

Fig. 2 simulation of (a) velocity, (b) microrotation, (c)temperature and (d) concentration.

The boundary conditions for $t>0$ :

$$
\begin{array}{r}
u(0, t)=\cos \omega t, \quad \theta(0, t)=t, \quad \phi=1, \\
u \rightarrow 0, \quad \Gamma=n \frac{\partial \Gamma}{\partial y}, \quad \theta \rightarrow 0, \quad \phi \rightarrow 0, \quad y \rightarrow \infty
\end{array}
$$

The shearing stress on the surface of a body, skinfriction, is defined as:

$$
\tau_{x}=(\mu+\kappa)\left(\frac{\partial u}{\partial y}\right)_{y=0}
$$

With the help of (11) the shearing stress component at the plate can be calculated in non-dimensional form as:

$$
(1-N) C_{f}=u^{\prime}(0)
$$

The local mass flux and the local Sherwood number are respectively given by

$$
\begin{aligned}
N u_{x} & =-x\left(\frac{\partial T}{\partial y}\right)_{y=0} /\left(T_{w}-T_{\infty}\right) \\
S h_{x} & =-x\left(\frac{\partial C}{\partial y}\right)_{y=0} /\left(C_{w}-C_{\infty}\right)
\end{aligned}
$$

We have dimenssionless form

$$
\begin{aligned}
N u_{x} R e^{-1} & =-\theta^{\prime}(0) \\
S h_{x} R e^{-1} & =-\phi^{\prime}(0)
\end{aligned}
$$

where $R e=\frac{v_{0} x}{\nu}$ is the Reynolds number.

\section{RESULTS AND DISCUSSION}

In order to get a physical view of the problem, numerical calculations are carried out for different values of chemical reaction, magnetic, radiation parameters and coupling number using pdepe.

Figure 2 show simulation for velocity, microrotation, temperature and concentration. The profiles of temperature and concentration decreases in a monotone fashion from the surface to a zero value far away

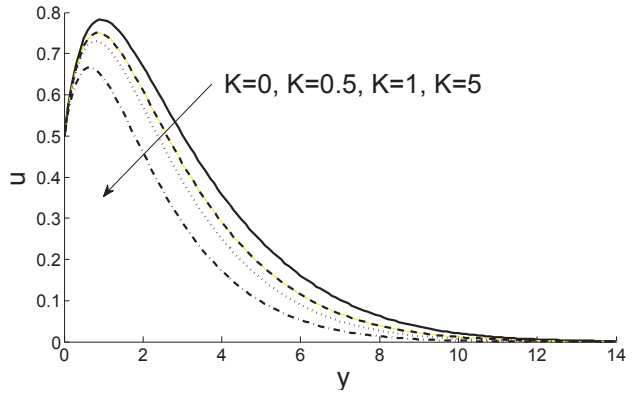

(a)

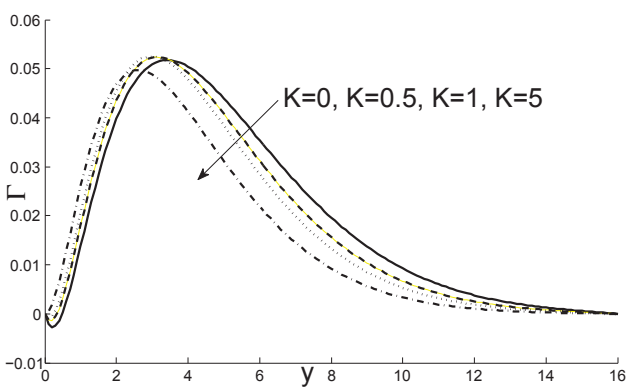

(b)

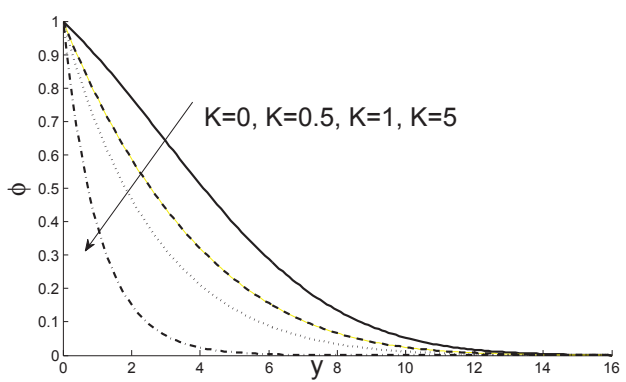

(c)

Fig. 3 Effect of chemical reaction parameter $K$ on velocity (u), microrotation $(\mathrm{G})$ and concentration (f) for $\mathrm{M}=5, \mathrm{Ra}=1, \mathrm{~N}=0.5$.

in the free stream for $K=1, \operatorname{Pr}=0.71, S c=0.22, R a=1, G c=$ $2, G r=2, M=5, N 1=0.01, N 2=5, t=0.2, N=0.5, n=$ $0, E c=0.001$ and $\omega=\pi / 6$ and these constants used throughout the discussion unless specified otherwise.

Figure 3 illustrates the effect of chemical reaction parameter $(K=$ $0,0.5,1,5)$, where $K=0$ corresponds the case no chemical reaction, on velocity angular velocity and concentration. As chemical reaction parameter increases both velocity and concentration decreases whereas microrotation increases inside the boundary layer and it decreases in the free stream. The hydrodynamic and concentration boundary layer thickness were observed to decrease as a result of increasing chemical reaction.

The presence of transverse magnetic field produces a resistive force (Lorentz force) on the fluid flow hence leads to slow down the motion of electrically conducting fluid. Fig. 4 it is seen that increasing the values of $M$ decreases the velocity and increases micrortation. It is also interesting to see that how the magnetic parameter affect the rotational behavior of $\Gamma$. Increasing the values magnetic parameter $M$ slightly decreases temperature inside the boundary layer. 


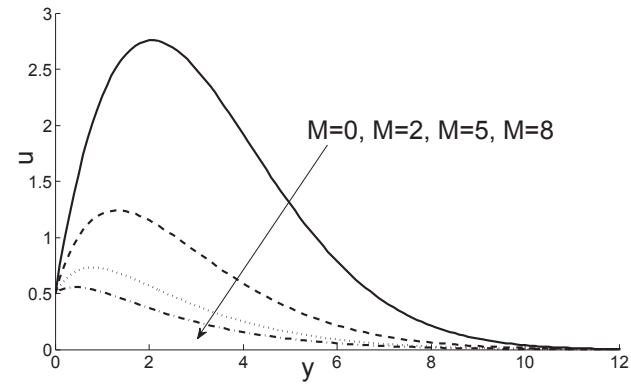

(a)

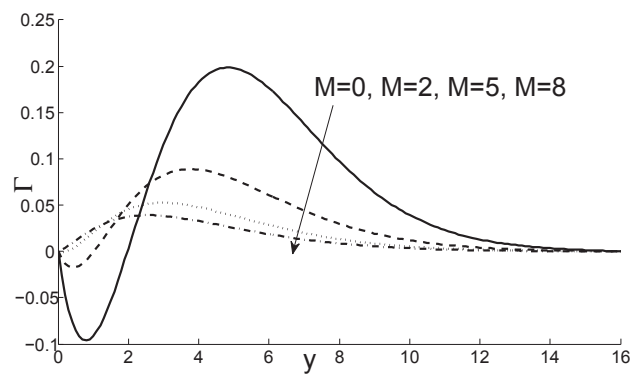

(b)

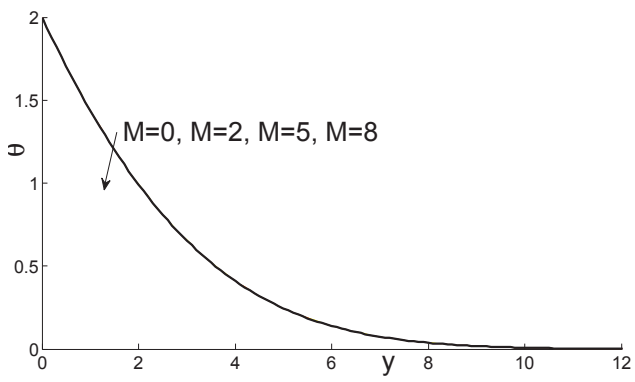

(c)

Fig. 4 Effect of magnetic parameter $M$ on velocity, microrotation (G) and concentration (f) for $\mathrm{K}=1, \mathrm{Ra}=1, \mathrm{~N}=0.5$.

It is marked from Fig. 5 that increasing value of radiation parameter leads to an increase in the velocity as well as temperature profiles where $R a=0$ corresponds absence of radiation. However, the reverse effect was seen for microrotation in its pick. The higher value of radiation parameter higher the thermal boundary-layer thickness.

Figure 6 represents the effects of coupling number $N$. When $N=0$, the fluid behaves as Newtonian fluid and equation (12) and (13) decoupled. Increasing the coupling number $N$ increases velocity, microrotation and temperature as depicted in Fig. 6 The case $N=0$ no microrotation shows in the graph of $\Gamma$. It is also noted that velocity of micropolar fluid greater than the velocity of Newtonian fluid. The effect of $N$ on temperature is found to be very slightly incrossing with $N$. The effect of $\omega$ on velocity shown in Fig. 6(d), as the phase angle increases the velocity decreases.

It is seen from the table $C f$ increases with an increasing in $R a, N$ but decreases with the $M, K$. The heat transfer at the plate, Nusselt number increases with the increase $M, R a, N$ it is also observed slight increase with $K$. The Sherwood number decreases with increase in the

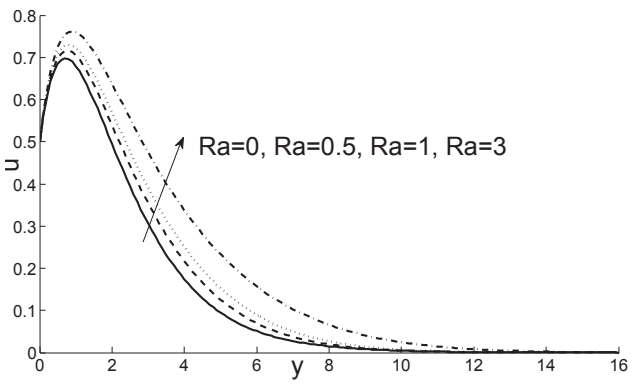

(a)

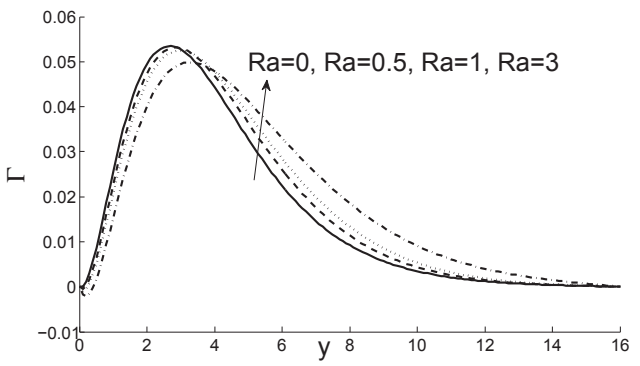

(b)

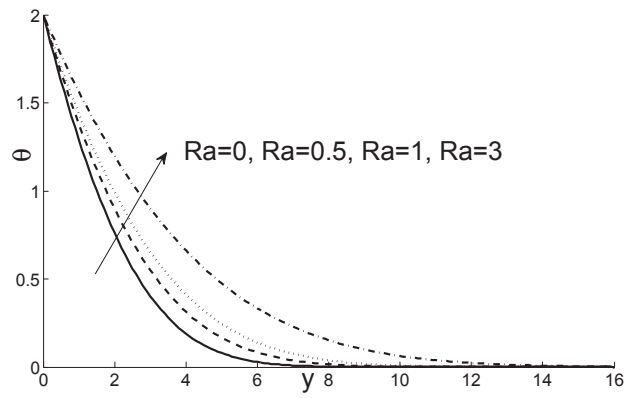

(c)

Fig. 5 Effect of radiation parameter $R a$ on velocity (u), microrotation (G) and temperature (?) for $\mathrm{M}=5, \mathrm{~K}=1, \mathrm{~N}=0.5$.

chemical reaction parameter but there is no sensitive influence with the various values of $M, N, R a$.

\section{CONCLUSIONS}

In this paper, investigations were made on the effects of radiation, magnetic, chemical reaction parameters and coupling numbers for chemically conducting unsteady micropolar fluid under the influence of magnetic field taking into account radiation for flow past an infinite vertical oscillating plate, with variable temperature. The results illustrate the flow characteristics for the velocity, microrotation, temperature, concentration, skin-friction, Nusselt number and Sherwood number. Magnetic parameter affects rotation component of the micropolar fluid. It is found that the velocity, microrotation, concentration as well as skin friction decreases with an increase in the chemical reaction parameter. The velocity and microration decreases with an increase in the magnetic parameter it is also seen the skin friction decreases when the magmatic parameter increases. Velocity and temperature increases with the radiation parameter. 


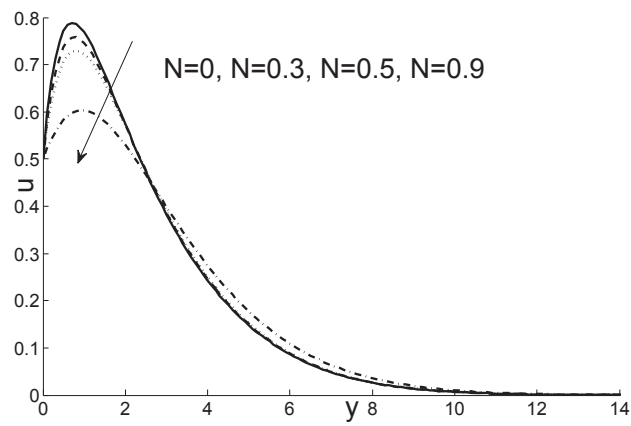

(a)

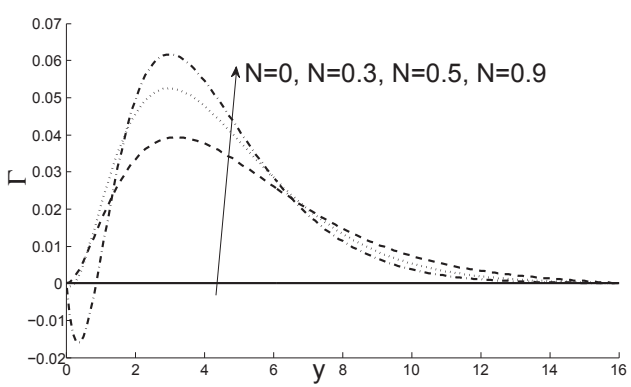

(b)

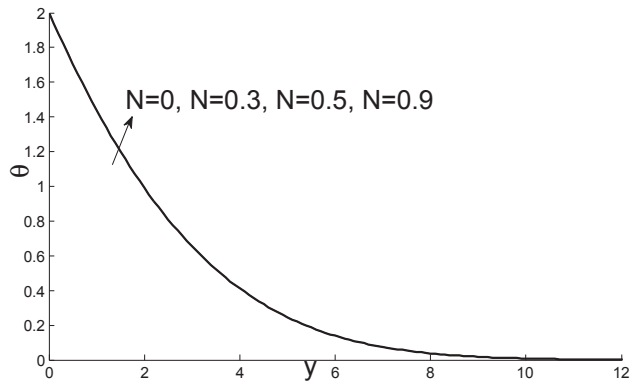

(c)

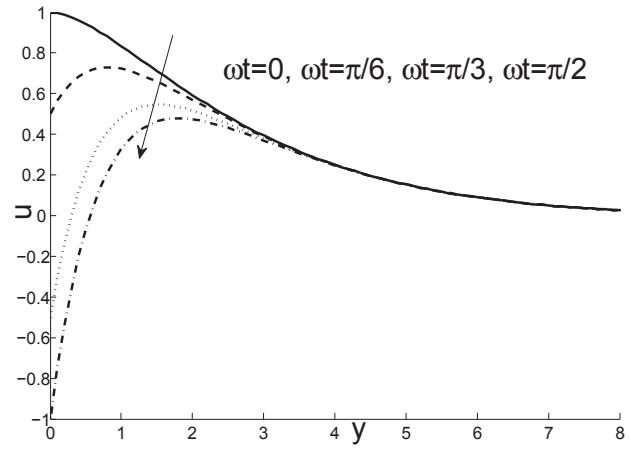

(d)

Fig. 6 Effect of coupling number $N$ on velocity (u), microrotation (G) and concentration (f) for $\mathrm{M}=5, \mathrm{~K}=1, \mathrm{Ra}=1$. The effect of $\omega t$ on velocity (d).
Table 1 The effects of the parameters $M, R a, N, K$ on the skin friction $C f$, Nusselt number $N u$, Sherwood number Sh for $G c=2, G r=$ $2, P r=0.71, S c=0.22, N 1=0.01, N 2=5$ and $E c=$ 0.001 .

\begin{tabular}{|cccc|ccc|}
\hline \hline $\mathrm{M}$ & $\mathrm{Ra}$ & $\mathrm{N}$ & $\mathrm{K}$ & $u^{\prime}(0)$ & $-\theta^{\prime}(0)$ & $-\phi^{\prime}(0)$ \\
\hline 0 & 1 & 0.5 & 1 & 20.8123 & 8.5978 & 18.2642 \\
1 & 1 & 0.5 & 1 & 19.6952 & 8.5979 & 18.2642 \\
5 & 1 & 0.5 & 1 & 16.6759 & 8.5982 & 18.2643 \\
8 & 1 & 0.5 & 1 & 15.2246 & 8.5985 & 18.2642 \\
\hline 5 & 0 & 0.5 & 1 & 16.6046 & 8.0808 & 18.2643 \\
5 & 0.5 & 0.5 & 1 & 16.6479 & 8.4045 & 18.2643 \\
5 & 1 & 0.5 & 1 & 16.6759 & 8.5982 & 18.2643 \\
5 & 3 & 0.5 & 1 & 16.7347 & 8.9738 & 18.2642 \\
\hline 5 & 1 & 0.1 & 1 & 16.1142 & 8.5980 & 18.2643 \\
5 & 1 & 0.5 & 1 & 16.6759 & 8.5982 & 18.2643 \\
5 & 1 & 0.7 & 1 & 17.1016 & 8.5983 & 18.2643 \\
5 & 1 & 0.9 & 1 & 17.8789 & 8.5984 & 18.2642 \\
\hline 5 & 1 & 0.5 & 0 & 16.7669 & 8.5982 & 18.8796 \\
5 & 1 & 0.5 & 0.5 & 16.7192 & 8.5982 & 18.5585 \\
5 & 1 & 0.5 & 1 & 16.6759 & 8.5982 & 18.2643 \\
5 & 1 & 0.5 & 5 & 16.4294 & 8.5984 & 16.5404 \\
\hline
\end{tabular}

\section{NOMENCLATURE}

$G_{r} \quad$ Grashof number

$M \quad$ magnetic parameter

$P_{r} \quad$ Prandtl number

$G_{c} \quad$ modified Grashof number

$K \quad$ non-dimensional chemical reaction parameter

$R a \quad$ radiation parameter

$c_{p} \quad$ specific heat

$C_{f} \quad$ local skin friction coefficient

$T \quad$ temperature of the fluid

$T_{w} \quad$ temperature at the wall

$T_{\infty} \quad$ temperature of the fluid far away from the wall

$C_{\infty} \quad$ concentration of the fluid far away from the wall

$C_{w} \quad$ concentration at the wall

$k \quad$ thermal conductivity

$T \quad$ temperature of the fluid

$C \quad$ concentration of the fluid

$g$

$K_{1}$

$B_{0}$ gravitational acceleration chemical reaction parameter magnetic ?eld intensity effective diffusive coefficient velocity component in $\mathrm{x}$-direction velocity component in $\mathrm{y}$-direction velocity of the plate

Eckert number

Schmidt number suction velocity coordinate coordinate

Greek Symbols

$\mu \quad$ the dynamic viscosity

$\beta_{T} \quad$ thermal expansion coefficient

$\beta_{C} \quad$ concentration expansion coefficient

$\alpha \quad$ thermal diffusivity

$\nu \quad$ kinematic viscosity

$\rho \quad$ density of the fluid

$\tau_{w} \quad$ wall shear stress

$\Gamma \quad$ microrotation component

$\sigma \quad$ fluid electrical conductivity 


$\begin{array}{ll}\gamma & \text { viscosity coefficient } \\ \kappa & \text { material constant (viscosity coefficient) } \\ \rho & \text { density } \\ \sigma * & \text { Stefan-Boltzmann constant }\end{array}$

\section{REFERENCES}

Ahmed, N., Sarma, D., and Deka, H., 2012, "MHD Mixed Convection and Mass Transfer from an Infinite Vertical Porous Plate with Chemical Reaction in Presence of a Heat Source," Applied Mathematical Sciences, 6(21), 1011-1020.

Ariman, T., Turk, M., and Sylvester, N., 1990, "Review ArticleApplications of Microcontinuum Fluid Mechanics," International Journal of Engineering Science, 12, 273-293.

http://dx.doi.org/10.1016/0020-7225(74)90059-7.

Chaudhary, R.C., and Jha, A.K., 2008, "Effects of Chemical Reactions on MHD Micropolar Fluid Flow Past a Vertical Plate in Slip-Flow Regime," Applied Mathematics and Mechanics, 29(9), 1179-1194.

http://dx.doi.org/10.1007/s10483-008-0907-x.

Eringen, A.C., 1966, “Theory of Micropolar Fluids," Journal of Mathematics and Mechanics, 16, 1-8.

Hossain, M.A., Hussain, S., and Rees, D.A.S., 2001, "Influence of Fluctuating Surface Temperature and Concentration on Natural Convection Flow from a Vertical Flat Plate," Zeitschrift Fur Angewandte Mathematik Und Mechanik, 81(10), 699-709.

http://dx.doi.org/10.1002/1521-4001(200110)81:10<699::AID-

ZAMM699>3.0.CO;2-3.

Hsiao, K.L., 2010, "Heat and Mass Mixed Convection for MHD ViscoElastic Fluid Past a Stretching Sheet with Ohmic Dissipation," Communications in Nonlinear Science and Numerical Simulation, 15, 1803-1812.

Jain, N.C., Chaudhary, D., and Dinesh, K.V., 2012, “Unsteady Three Dimensional Free Convection Heat and Mass Transfer Flow Embedded in a
Porous Medium with Periodic Permeability and Constant Heat and Mass Flux," Applied Mathematics, 2(3), 70-76. http://dx.doi.org/10.5923/j.am.20120203.05.

Muthucumaraswamy, R., and Meenakshisundaram, S., 2006, "Theoretical Study of Chemical Reaction Effects on Vertical Oscillating Plate with Variable Temperature," Theoretical and Applied Mechanics, 33(3), 245257.

http://dx.doi.org/10.2298/TAM0603245M.

Muthucumaraswamy, R., and P, G., 2002, "Natural Convection on a Moving Isothermal Vertical Plate with Chemical Reaction," Journal of Engineering Physics and Thermophys, 75(1), 113-119.

http://dx.doi.org/10.1023/A:1014826924926.

Patrick, H.O., and Paul, J.T., 2010, "Natural Conductive Heat Transfer from a Narrow Vertical Flat Plate with a Uniform Surface Heat Flux and with Different Plate Edges Conditions," Frontiers in Heat and Mass transfer, 1, 300-306.

http://dx.doi: 10.5098/hmt.v1.1.3006 .

Rahman, M., and Mulolani, I., 2000, "Convective Diffusive Transport Chemical Reaction in Natural Convection Flows," Theoretical and Computational Fluid Dynamics, 13(5), 291-304. http://dx.doi.org/10.1007/s001620050001.

Soundalgekar, V.M., and Wavre, P.D., 1997, "Unsteady Free Convection Flow Past an Infinite Plate with Variable Suction and Mass Transfer," International Journal of Heat and Mass Transfer, 20(12), 1375-1380. http://dx.doi.org/10.1016/0017-9310(77)90034-5.

Srinivasacharya, D., and Kaladhar, K., 2014, "Effects of Thermal and Soutal Stratification on mixed Convection Flow Along a Vertical Plate Saturated with Couple Stress Fluid," Frontiers in Heat and Mass Transfer, $5-11$.

http://dx.doi.org/10.5098/hmt.5.11. 